\title{
Effects of long-term soft contact lens wear on corneal epithelial thickness after small incision lenticule extraction
}

\author{
YUN WANG ${ }^{1}$, XIAOFENG ZHANG ${ }^{1,2}$, XIAOLONG YANG $^{2}$, YUE XU $^{2}$, BAOGEN LUO $^{2}$ and YIFENG QIAN ${ }^{2}$ \\ ${ }^{1}$ Department of Ophthalmology, Suzhou Dushu Lake Hospital (Dushu Lake Hospital Affiliated Soochow University, \\ Medical Center of Soochow University), Suzhou, Jiangsu 215100; ${ }^{2}$ Department of Ophthalmology, \\ The First Affiliated Hospital of Soochow University, Suzhou, Jiangsu 215006, P.R. China
}

Received January 19, 2020; Accepted September 27, 2021

DOI: $10.3892 /$ etm.2021.10930

\begin{abstract}
The present study investigated changes in corneal epithelial thickness after small incision lenticule extraction (SMILE) in patients with long-term preoperative soft contact lens (SCL) wear, the impact of SCL wear on the efficacy of surgical outcomes and the effects of long-term SCL wear on postoperative corneal aberrations. Patients were assigned to three groups according to the duration of SCL wear: Group A, the non-SCL-wearing group; group B, those with SCL wear $\leq 1$ year; and group C, those with SCL wear $>1$ year. Epithelial thickness was recorded in nine zones by anterior segment optical coherence tomography across a 5-mm diameter before surgery and at 1 week, and 1 , 3 and 6 months post-surgery. Corneal epithelial thickness and corneal aberrations among the three groups were compared, as well as the effects of changes in corneal epithelial thickness on postoperative visual acuity and manifest refraction. No significant differences were noted with regard to age or preoperative spherical equivalent among groups A (22 eyes), B (17 eyes) and $\mathrm{C}$ (18 eyes). Preoperative corneal epithelial thickness in the inferonasal, inferior and inferotemporal zones was thinner in group B compared with that in group A, and corneal epithelial thickness was thinner in all nine zones in group $\mathrm{C}$ compared with that in group $\mathrm{A}(\mathrm{P}<0.05)$. At all follow-up time points, in the central, nasal, inferonasal, inferior, inferotemporal and temporal areas, the epithelial thickness was thinner in group $\mathrm{C}$ compared with that in group $\mathrm{A}(\mathrm{P}<0.05)$. At 3 months postoperatively, the epithelial thickness was thinner in the
\end{abstract}

Correspondence to: Professor Xiaofeng Zhang, Department of Ophthalmology, The First Affiliated Hospital of Soochow University, 188 Shizi Road, Suzhou, Jiangsu 215006, P.R. China E-mail: zhangxiaofeng@suda.edu.cn

Abbreviations: SMILE, small incision lenticule extraction; SCL, soft contact lens; UDVA, uncorrected distance visual acuity; IOP, intraocular pressure; SD, standard deviation

Key words: SCL, SMILE, corneal epithelial thickness, corneal aberration, optical coherence tomography inferonasal and inferior sectors in group $\mathrm{C}$ compared with that in group $\mathrm{B}(\mathrm{P}<0.05)$, and at 6 months postoperatively, the epithelial thickness in the inferior region was thinner in group $\mathrm{C}$ compared with that in group $\mathrm{B}(\mathrm{P}<0.05)$. There were no significant differences in visual acuity or manifest refraction among the three groups at all postoperative time points. The total higher-order aberrations were greater in group $\mathrm{C}$ compared with those in group $\mathrm{A}$ for all time points $(\mathrm{P}<0.05)$ and were greater in group $\mathrm{C}$ at 1 and 3 months postoperatively compared with those in group $\mathrm{B}(\mathrm{P}<0.05)$. The spherical aberrations at 3 and 6 months postoperatively were greater in group $\mathrm{C}$ compared with those in group $\mathrm{A}(\mathrm{P}<0.05)$. The coma aberrations were greater in group $\mathrm{C}$ compared with those in groups $\mathrm{A}$ and $\mathrm{B}$ for all time points $(\mathrm{P}<0.05)$. In conclusion, long-term SCL wear will result in corneal epithelial thinning, which does not impact visual acuity or manifest refraction after SMILE.

\section{Introduction}

The number of teenagers who exhibit myopia and myopic astigmatism is increasing each year. Numerous patients choose soft contact lenses (SCLs) to manage the problem of refractive error. The normal physiology of the ocular surface $(1,2)$, including changes in the corneal epithelium $(3,4)$ and endothelium $(5,6)$, can be affected by direct contact between the contact lens and the cornea. Numerous previous studies have suggested that long-term SCL wear can decrease oxygen uptake (1), induce cell death (7) and lead to corneal epithelial thinning (8-10), which may become more obvious with extended SCL wear. The corneal epithelial thickness can increase following discontinuation of SCL wear; however, the corneal epithelial thickness is unable to reach the normal range exhibited by people without SCL wear (11). A previous study (12) demonstrated that the corneal epithelial thickness reached stability following discontinued wear of SCLs within 2 weeks; nevertheless, individual variation should not be discounted.

In recent years, methods of corneal refractive surgery have been improving. Corneal refractive surgery provided another option for the correction of visual acuity, particularly in myopic patients with the experience of SCL wear. Small incision lenticule extraction (SMILE) is a relatively new surgical 
method of corneal refractive surgery, which realizes the flapless surgical mode, leaving the epithelium and the Bowman's layer undamaged except for the small side cut incision to extract the lenticule (13). After SMILE, corneal biomechanical properties exhibit greater stability. Reinstein et al (14) demonstrate that the postoperative total stromal tensile strength following SMILE is higher than both photorefractive keratecotomy (PRK) and laser in situ keratomileusis (LASIK). Subepithelial stromal plexus damage and the postoperative inflammatory reaction are decreased and dry eye symptoms are less pronounced $(15,16)$. This surgical method has advantages in terms of safety, effectiveness, good predictability and stability $(13,17)$. Numerous myopic patients with experience of wearing SCLs have chosen corneal refractive surgery to obtain satisfactory visual acuity. The purpose of the present study was to investigate epithelial thickness changes after SMILE following long-term wear of SCLs, as well as the influence of SCL wear on surgical outcomes.

Previous studies have been performed to evaluate the central region of the cornea $(18,19)$. In the present study, anterior segment optical coherence tomography imaging was applied to determine corneal epithelial thickness in nine zones across a 5-mm corneal diameter, which demonstrated higher reproducibility and accuracy in both normal and postoperative eyes.

\section{Patients and methods}

Patients. This retrospective observational study included 57 eyes of 30 patients (16 male and 14 female; mean age 25.90 \pm 5.90 years; range 16-34 years) who were scheduled for SMILE as treatment for myopia or myopia astigmatism at The First Affiliated Hospital of Soochow University (Suzhou, China) between June 2017 and April 2018. A complete ophthalmic examination was performed to screen for corneal abnormalities and determine the eligibility of the patients for refractive surgery. The inclusion criteria were: Age $>18$ years; spherical equivalent refraction converted by preoperative optometry of -2.00 to -6.00 diopters; intraocular pressure ranging from 10-21 mmHg; no history of hard contact lens wear; and a normal corneal topograghy examination. Patients with ocular pathological conditions such as corneal opacity and keratoconus were excluded. Additionally, patients with other ocular diseases and systemic organic diseases affecting the recovery from surgery were excluded from the study.

A previous study found that a 2 -week contact lens-free period seemed to be adequate for the cornea to stabilize (9). The present study aimed to elucidate the effects of long-term SCL wear on corneal epithelial thickness; therefore, 1 year was chosen as the long-term period. According to the duration of SCL wear, the patients were divided into three groups. Patients who had never worn SCLs were included into group A, while the others who had worn SCLs for $<1$ year were in group B and those who had worn SCLs for $>1$ year were in group C. All patients were asked to stop wearing SCLs at least 2 weeks prior to the surgery. This retrospective study was approved by the Ethics Committee of The First Affiliated Hospital of Soochow University, and all patients provided consent at the time of the surgery.

Corneal epithelial thickness measurement. The corneal epithelial thickness data were obtained preoperatively and then at 1 week, and 1, 3 and 6 months postoperatively using the RTVue-100 anterior segment optical coherence tomography system (Optovue, Inc.). Each measurement was performed by one experienced technician in a room where light levels were reduced by half. All patients were forbidden from using any eye drops for $2 \mathrm{~h}$ prior to the measurements.

The anterior segment optical coherence tomography obtained the epithelial thickness of a circular region over a 6-mm diameter centered at the pupil center. The maps were divided into 3 zones by the annular rings: Central zone (2-mm diameter), paracentral zone (2- to 5-mm diameter) and mid-peripheral zone (5- to 6-mm diameter). The paracentral and mid-peripheral zones were each divided into the following 8 sectors: Superior, superonasal, nasal, inferonasal, inferior, inferotemporal, temporal and superotemporal (Fig. 1). Finally, epithelial maps were created and left eyes maps were mirrored vertically so as to make them comparable with right eyes. The present study analyzed the corneal epithelial thickness of 9 sectors over the central and paracentral zones, as well as the maximum-minimum (max-min) and standard deviation (SD) values of corneal epithelial thickness in the central 5 -mm diameter (due to the covering of eyelids, peripheral data were not accurate.).

Corneal topography measurement. Corneal topography measurements were acquired using a Sirius Corneal Topographer (CSO, Inc.). Three consecutive scans were taken and the best quality scan was selected for further analysis. For this, the Scheimpflug image area needed to be $>90 \%$, the centration positioning needed to be $>90 \%$ and the Placido disk needed to cover an area of $>90 \%$. The total higher order aberrations, spherical aberrations and coma aberrations were noted for analysis.

Surgical technique. All myopic operations were performed by one experienced surgeon (from Department of Ophthalmology, The First Affiliated Hospital of Soochow University). Preoperative medication included topical $0.5 \%$ levofloxacin (Santen Pharmaceutical Co., Ltd.) three times for 3 days.

All SMILE procedures were performed by VisuMax $500-\mathrm{kHz}$ femtosecond laser (Carl Zeiss AG). The diameter of the cap was 7.3-7.5 $\mathrm{mm}(7.48 \pm 0.05 \mathrm{~mm})$ and the intended cap thickness was $120 \mu \mathrm{m}$. A $3-\mathrm{mm}$ incision was made at the $90^{\circ}$ meridian to extract the lenticule and the side-cut angle was $90^{\circ}$. The optical zone diameter was $6.3-6.8 \mathrm{~mm}$ $(6.60 \pm 0.13 \mathrm{~mm})$. The maximum thickness of the lenticule was 59-135 $\mu \mathrm{m}(108.5 \pm 17.9 \mu \mathrm{m})$ and the lenticule side-cut angle was $90^{\circ}$. The data were described as mean \pm SD.

Postoperative medications included topical tobramycin dexamethason (Novartis International AG) four times for $30 \mathrm{~min}$ after surgery, $0.5 \%$ levofloxacin (Santen Pharmaceutical Co., Ltd.) four times every day for 7 days, $0.1 \%$ fluorometholone (Santen Pharmaceutical Co., Ltd.) four times every day, which decreased one time each week until once a day for one week. Patients were followed up at postoperative week 1 , and months 1, 3 and 6 . Uncorrected distance visual acuity (UDVA), manifest refraction and intraocular pressure (IOP) were measured, and anterior segment optical coherence tomography and corneal topography were performed at every visit. 


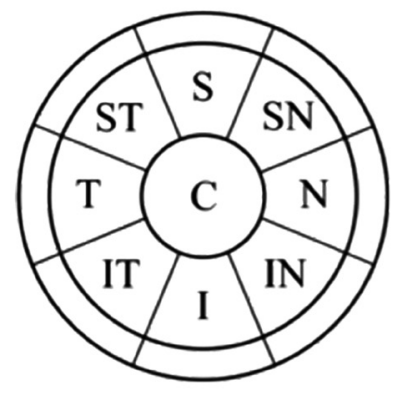

OD

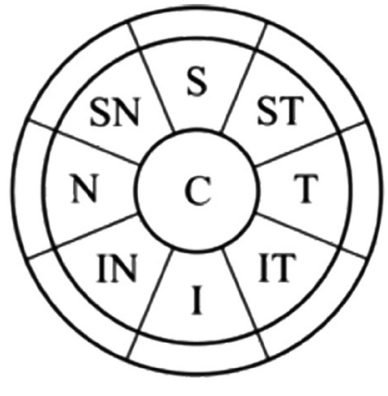

OS
Figure 1. Zoning of corneal epithelium (diameter of central $6 \mathrm{~mm}$ ). C, central; S, superior; SN, superonasal; N, nasal; IN, inferonasal; I, inferior; IT, inferotemporal; T, temporal; ST, superotemporal; OD, oculus dexter; OS, oculus sinister.

Statistical analysis. SPSS for Windows software (version 17.0; SPSS, Inc.) was used for statistical analysis. Preoperative examinations among groups were analyzed by one-way ANOVA followed by Bonferroni's correction. The corneal aberrations within groups, the epithelial thickness and the difference in epithelial thickness, the corneal aberrations and the difference in corneal aberrations and the max-min and SD values of corneal epithelial thickness across a 5-mm diameter among three groups were compared with a two-way mixed ANOVA followed by Bonferroni's correction. Kruskal-Wallis $\mathrm{H}$ test was used to analyze UDVA and spherical equivalent among three groups. $\mathrm{P}<0.05$ was considered to indicate a significant difference.

\section{Results}

Study population and characteristics. The study cohort consisted of 30 patients with 57 eyes undergoing SMILE for myopia correction. A total of 8 eyes were treated in group B in patients who had worn one-day disposable hydrogel SCLs (Johnson \& Johnson) and 9 eyes in patients who had worn monthly disposable hydrogel SCLs (Johnson \& Johnson or Bausch \& Lomb). A total of 8 eyes were treated in group C in patients who had worn one-day disposable hydrogel SCLs (Johnson \& Johnson) and 10 eyes in patients who had worn monthly disposable hydrogel SCLs (Johnson \& Johnson or Bausch \& Lomb). The ratio of daily and monthly disposable lens in group B was consistent with that in group $\mathrm{C}$. The mean age, preoperative spherical equivalent refractive power and preoperative epithelial thickness of the central 5-mm zone were similar among the three groups. There were no statistically significant differences in baseline measurements among the three groups, except epithelial thickness $(\mathrm{P}<0.05)$. The preoperative mean corneal epithelial thickness was thinner in group $\mathrm{C}$ than in group $\mathrm{A}(\mathrm{P}<0.05)$, whereas no significant difference in preoperative epithelial thickness was observed in group $\mathrm{B}$ compared with groups $\mathrm{A}$ and $\mathrm{C}(\mathrm{P}>0.05)$ (Table I).

Comparison of visual acuity and manifest refraction. Visual acuity values (in $\log$ MAR) at postoperative week 1 , and months 1, 3 and 6 were similar among the three groups. Analysis with the Kruskal-Wallis H test revealed no statistically significant differences in UDVA among the three groups at all postoperative time points $(\mathrm{P}>0.05$; Table II).

Spherical equivalent was compared at postoperative week 1 , and months 1, 3 and 6 among the three groups. Analysis with the Kruskal-Wallis $\mathrm{H}$ test revealed no statistically significant differences in spherical equivalent among the three groups at each pair of consecutive time points ( $\mathrm{P}>0.05)$ (Table II).

Epithelial thickness analysis. The preoperative superior epithelial thickness was thinner than the preoperative inferior epithelial thickness of the nine sectors in group $\mathrm{A}\left(F_{\mathrm{A}}=15.669\right.$, $\mathrm{P}<0.01 ; t=-2.614, \mathrm{P}<0.05)$; by contrast, there were no statistically significant differences in epithelial thickness of the nine sectors in groups $\mathrm{B}$ or $\mathrm{C}\left(F_{\mathrm{B}}=0.965, \mathrm{P}=0.395 ; F_{\mathrm{C}}=1.724\right.$, $\mathrm{P}=0.151)$. Preoperatively, the corneal epithelial thicknesses in the inferonasal, inferior and inferotemporal sectors were thinner in group B than in group A $(\mathrm{P}<0.05)$; the epithelial thicknesses of all nine sectors were thinner in group $\mathrm{C}$ than in group A ( $\mathrm{P}<0.05$; Fig. 2).

At postoperative week 1 and month 1, the epithelial thicknesses in the central, nasal, inferonasal, inferior, inferotemporal and temporal segments were thinner in group $\mathrm{C}$ than in group $\mathrm{A}(\mathrm{P}<0.05)$. At postoperative month 3 , the epithelial thicknesses in the central, nasal, inferonasal, inferior, inferotemporal and temporal regions were significantly thinner in group $\mathrm{C}$ than in group $\mathrm{A}(\mathrm{P}<0.05)$; the inferonasal and inferior regions were thinner in group $\mathrm{C}$ than in group $\mathrm{B}$. At postoperative month 6 , the epithelial thicknesses in the central, nasal, inferonasal, inferior, inferotemporal and temporal segments were significantly thinner in group $\mathrm{C}$ than in group $\mathrm{A}(\mathrm{P}<0.05)$; the epithelial thicknesses in the inferior segment were thinner in group C than in group B (Fig. 2).

In the superior and superonasal regions of the cornea, the change in epithelial thickness at postoperative week 1 and month 1 was greater in group $\mathrm{C}$ than in group $\mathrm{A}(\mathrm{P}<0.05)$ In the superotemporal region, the change in epithelial thickness was greater in group $\mathrm{C}$ than in group $\mathrm{A}$ at postoperative week 1 and month $1(\mathrm{P}<0.05)$; it was also greater in group $\mathrm{C}$ than in group $\mathrm{B}$ at postoperative week $1(\mathrm{P}<0.05$; Fig. 3$)$.

It should be noted that at all examined time points, the epithelial thickness in the central, nasal, inferonasal, inferior, inferotemporal and temporal segments was less in group $\mathrm{C}$ than in group A, but in the superior, superotemporal and superonasal zones had no statistically significant difference between group $\mathrm{A}$ and group $\mathrm{C}$ at all postoperative time points which the epithelial thickness difference of the three zones was more in group $\mathrm{C}$ than in group $\mathrm{A}$ at postoperative week 1 and month 1 (Fig. 2).

Uniformity of epithelial thickness. Table III shows the max-min values and SDs of epithelial thickness within the central 5-mm zone for the examined time points, compared among the three groups. Statistically significant differences were present in the max-min values among the three groups at postoperative week 1 , month 3 and $6(\mathrm{P}<0.05)$. Differences were also observed in SD values among the three groups at all postoperative time points except postoperative month 1; the max-min value and SD in group $\mathrm{C}$ were lower than those in group $\mathrm{A}$ at postoperative week 1 , month 3 and $6(\mathrm{P}<0.05$; Table III). 
Table I. Preoperative comparative data for all three study groups ${ }^{\mathrm{a}}$.

\begin{tabular}{|c|c|c|c|c|c|}
\hline Parameters & Group A & Group B & Group C & F-value & P-value \\
\hline Eyes, n & 22 & 17 & 18 & - & - \\
\hline Age, years & $23.55 \pm 6.46$ & $26.78 \pm 5.07$ & $28.30 \pm 4.16$ & 2.156 & 0.135 \\
\hline Sphere, D & $-4.25 \pm 1.10$ & $-4.43 \pm 1.12$ & $-4.61 \pm 1.15$ & 0.511 & 0.603 \\
\hline Cylinder, Dcyl & $-0.80 \pm 0.37$ & $-0.75 \pm 0.40$ & $-0.57 \pm 0.55$ & 1.407 & 0.254 \\
\hline SE, D & $-4.65 \pm 1.11$ & $-4.80 \pm 1.13$ & $-4.90 \pm 1.08$ & 0.259 & 0.772 \\
\hline $\mathrm{ET}, \mu \mathrm{m}$ & $57.10 \pm 2.90$ & $55.52 \pm 2.59$ & $54.05 \pm 1.71$ & 7.462 & $0.001^{\mathrm{b}}$ \\
\hline
\end{tabular}

${ }^{a}$ Values expressed as mean \pm standard deviation. ${ }^{b} \mathrm{P}<0.01$; one-way ANOVA (Bonferroni's correction). Group A, non-wearing SCL; group B, wearing SCL $\leq 1$ year; group C, wearing SCL >1 year; D, diopters; Dcyl, diopters cylinder; SE; spherical equivalent; ET, corneal epithelial thickness; SCL, soft contact lens.

Table II. Comparative data of UDVA and SE among three groups ${ }^{\mathrm{a}}$.

\begin{tabular}{llcrrrr}
\hline Parameters & \multicolumn{1}{c}{ Time } & Group A $(\mathrm{n}=22)$ & Group B $(\mathrm{n}=17)$ & Group C (n=18) & $\mathrm{U}$ & P-value \\
\hline UDVA, logMAR & 1 week postoperative & $-0.05 \pm 0.06$ & $-0.05 \pm 0.06$ & $-0.04 \pm 0.06$ & 0.402 & 0.818 \\
& 1 month postoperative & $-0.06 \pm 0.06$ & $-0.05 \pm 0.06$ & $-0.06 \pm 0.07$ & 0.475 & 0.789 \\
& 3 months postoperative & $-0.05 \pm 0.05$ & $-0.04 \pm 0.08$ & $-0.04 \pm 0.04$ & 0.142 & 0.932 \\
& 6 months postoperative & $-0.09 \pm 0.07$ & $-0.07 \pm 0.06$ & $-0.04 \pm 0.07$ & 4.018 & 0.134 \\
SE, D & 1 week postoperative & $0.05 \pm 0.30$ & $0.07 \pm 0.49$ & $-0.20 \pm 0.43$ & 2.978 & 0.226 \\
& 1 month postoperative & $0.01 \pm 0.42$ & $0.10 \pm 0.39$ & $0.19 \pm 0.26$ & 1.696 & 0.428 \\
& 3 months postoperative & $0.03 \pm 0.35$ & $0.05 \pm 0.37$ & $-0.13 \pm 0.46$ & 1.637 & 0.441 \\
& 6 months postoperative & $0.02 \pm 0.28$ & $0.05 \pm 0.49$ & $0.02 \pm 0.28$ & 0.103 & 0.950 \\
\hline
\end{tabular}

${ }^{\text {a}}$ Values expressed as mean \pm standard deviation. Kruskal-Wallis H test. Group A, non-wearing SCL; group B, wearing SCL $\leq 1$ year; group C, wearing SCL >1 year; SCL, soft contact lens; UDVA, uncorrected distance visual acuity; SE, spherical equivalent.

Corneal aberrations analysis. The total higher-order aberrations of the three groups were increased postoperatively, compared with preoperatively $(\mathrm{P}<0.05)$. Preoperatively and at postoperative months 1,3 and 6 , the total higher-order aberrations were greater in group $\mathrm{C}$ than in group $\mathrm{A}(\mathrm{P}<0.05)$. At postoperative month 1 and 3 , the total higher-order aberrations were greater in group $\mathrm{C}$ than group $\mathrm{B}(\mathrm{P}<0.05)$. However, there were no statistically significant differences in the numbers of total higher-order aberrations difference among the three groups after surgery $(\mathrm{P}>0.05)$.

Postoperatively, spherical aberrations were increased among the three groups, compared with preoperative measurements $(\mathrm{P}<0.05$; Table IV). Preoperatively and at postoperative month 1 , no statistically significant differences were found in spherical aberrations among the three groups $(\mathrm{P}>0.05)$. However, at postoperative months 3 and 6 , spherical aberrations were greater in group $\mathrm{C}$ than group $\mathrm{A}(\mathrm{P}<0.05)$. At postoperative 1 month, the change in spherical aberrations was greater in group $\mathrm{C}$ than in group $\mathrm{A}(\mathrm{P}<0.05)$. At all time points postoperatively, the change in spherical aberrations was greater in group $\mathrm{C}$ than in groups $\mathrm{A}(\mathrm{P}<0.05)$.

Coma aberrations in group $\mathrm{A}$ at postoperative month 3 , in group B at postoperative month 6 , as well as in group $\mathrm{C}$ at postoperative at all time points, were greater than preoperative values $(\mathrm{P}<0.05$; Table IV). At all examined time points, coma aberrations were greater in group $\mathrm{C}$ than in groups $\mathrm{A}$ and $\mathrm{B}$ $(\mathrm{P}<0.05)$. Postoperative changes in coma aberrations tended to differ among the three groups, although these differences were not statistically significant ( $\mathrm{P}>0.05$; Table IV; Fig. 4).

No postoperative complications (e.g., refraction regression, epithelial defects or diffuse lamellar keratitis) were observed during the first 6 months postoperatively in any of the eyes.

\section{Discussion}

Previous studies have demonstrated that contact lens wear can lead to corneal epithelial thinning $(8,11)$. The results of the present study showed that corneal epithelial thicknesses were thinner in the short-term wear group than in the non-wearing group only in the inferonasal, inferior and inferotemporal sectors. The epithelial thicknesses were thinner in the long-term wear group compared with those in the non-wearing group in all nine sectors over the 5-mm diameter area. This indicated that longer contact lens wear was associated with a greater number of sectors with thinner corneal epithelial thickness.

Preoperatively, the corneal epithelial thickness is not evenly distributed; it is characterized by a thinner epithelium superiorly compared with that inferiorly (20), as confirmed in the present study. By contrast, no differences were found 
A

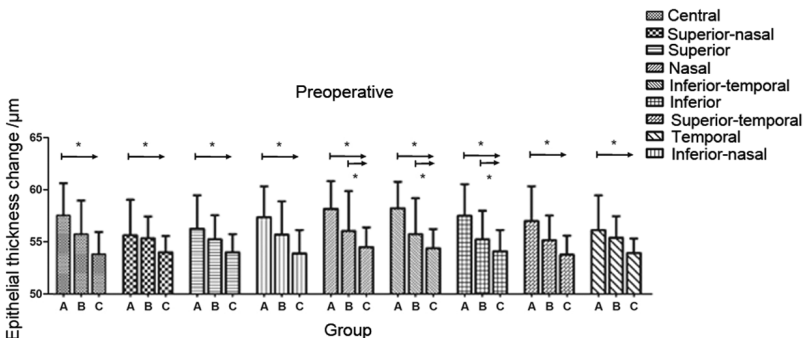

B

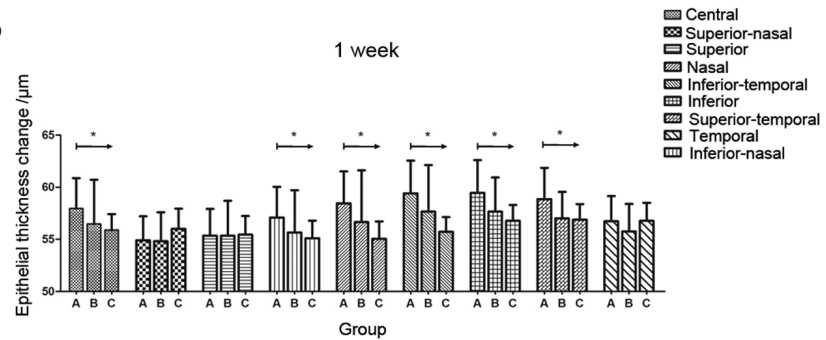

C

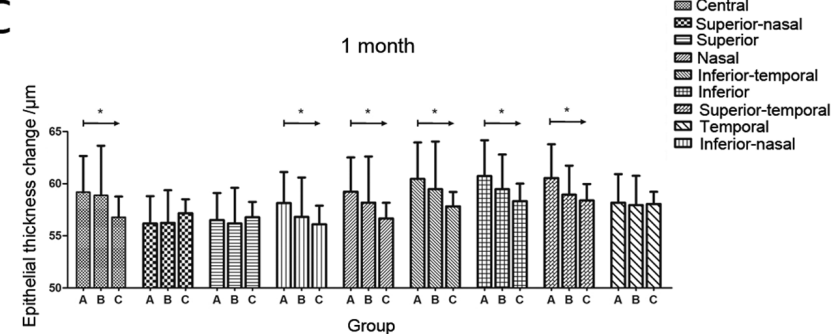

D

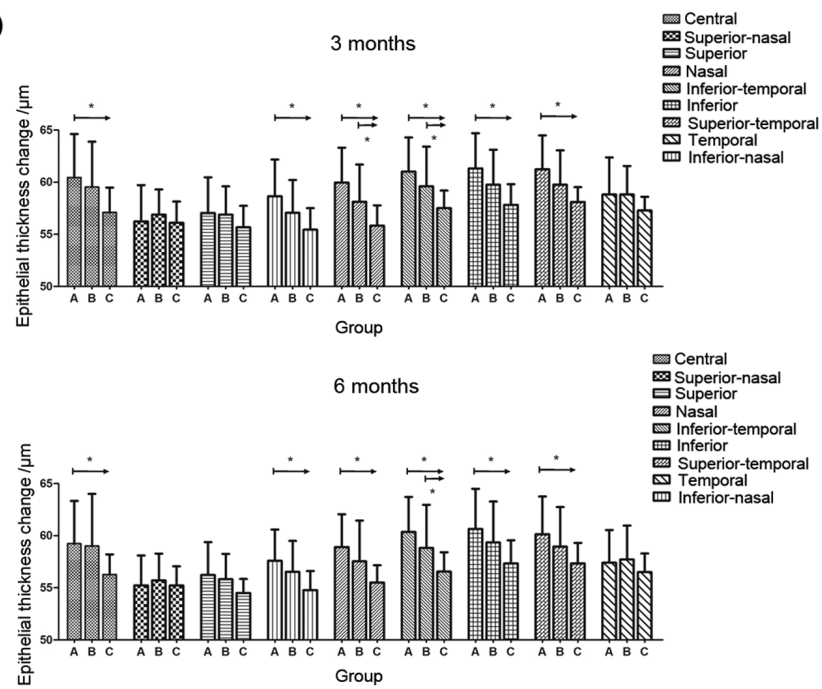

Figure 2. Corneal epithelial thickness in (A) preoperative, (B) 1 week, (C) 1 month, (D) 3 month and (E) 6 month of group A (non-SCL-wearing), group $\mathrm{B}$ (wearing $\mathrm{SCL} \leq 1$ year) and group $\mathrm{C}$ (wearing $\mathrm{SCL}>1$ year; ${ }^{*} \mathrm{P}<0.05$ ). Two-way ANOVA (Bonferroni's correction). SCL, soft contact lens.

in epithelial thicknesses in different segments between the short- and long-term wear groups. Corneal epithelial remodeling due to contact lens wear may have resulted in a uniform distribution of corneal epithelial thickness. The max-min and SD values of corneal epithelial thickness were used to describe the uniformity of the corneal epithelial distribution, using a previously published method (21). The present results showed that the max-min and SD values were lower in the long-term wear group than those in the non-wearing group, both before and after surgery; these findings indicated uniform corneal epithelium distribution
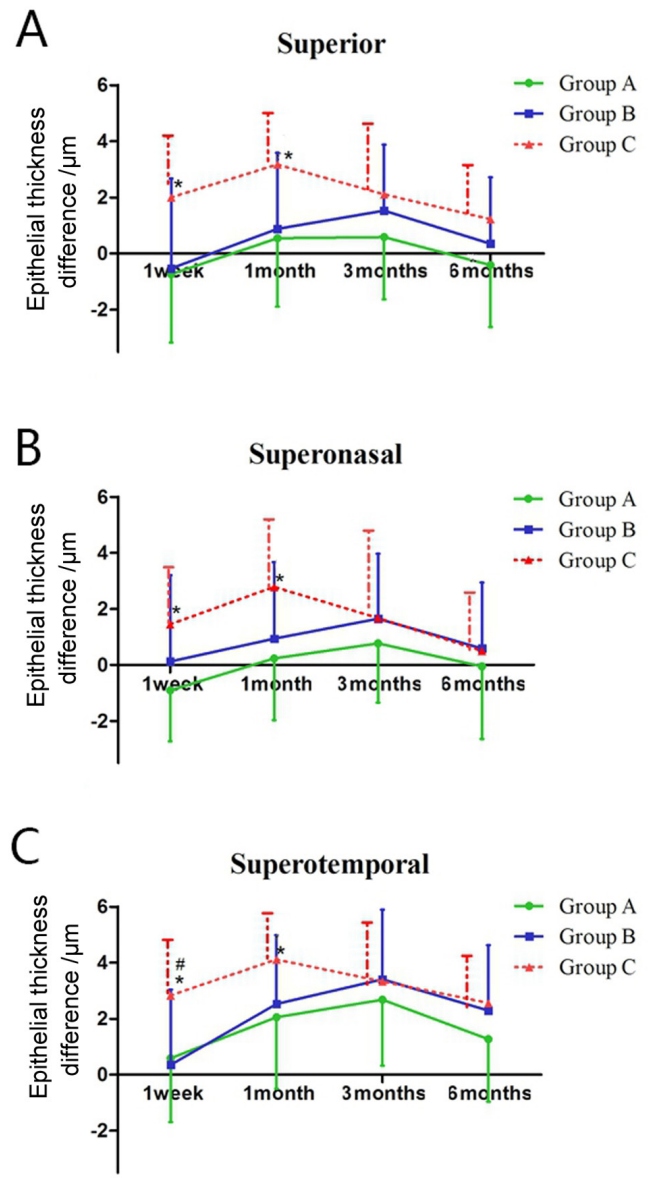

Figure 3. Corneal epithelial thickness difference in (A) superior, (B) superonasal and (C) superotemporal sectors of group A (non-SCL-wearing), group B (wearing $\mathrm{SCL} \leq 1$ year) and group $\mathrm{C}$ (wearing $\mathrm{SCL}>1$ year) preoperatively and at postoperative week 1 , and months 1,3 and $6\left({ }^{*} \mathrm{P}<0.05\right.$, comparing between group $\mathrm{A}$ and group $\mathrm{C}$; ${ }^{\text {"}} \mathrm{P}<0.05$, comparing between group $\mathrm{B}$ and group $\mathrm{C}$ ). Two-way mixed ANOVA (Bonferroni's correction). SCL, soft contact lens.

due to corneal epithelial remodeling after contact lens wear. The results were consistent with the observations of Lei et al (11) and Pérez et al (22).

The underlying mechanisms of corneal epithelial thinning after contact lens wear have been investigated in multiple studies. Corneal oxygen uptake plays an important role in corneal metabolism and the maintenance of corneal transparency (23). The oxygen required for corneal metabolism is derived from the oxygen that diffuses through the tear film, as well as oxygen supplied by the limbal vascular circulation. Contact lens wear affects corneal oxygen uptake (1). Patel et al (24) used confocal microscopy to study the corneal epithelium in vivo. The study reported that epithelial cells in long-term contact lens wearers became enlarged and exhibited a loose arrangement; moreover, the density of epithelial basal cells was decreased compared with that of non-contact lens wearers. The observed decrease in epithelial cell number and the thinning of the corneal epithelium are mainly caused by corneal hypoxia. Based on animal experiments, Yanai et al (4) suggested that hypoxia of the ocular surface led to disruption of tight junctions between corneal epithelial cells. In addition, mechanical stimulation of the ocular surface, due to the physical presence of contact lenses, 
Table III. Comparative data of corneal epithelial map within central 5-mm uniformity indices among three groups $\mathrm{s}^{\mathrm{a}}$.

\begin{tabular}{|c|c|c|c|}
\hline Parameters & Group A $(n=22)$ & Group B (n=17) & Group C $(n=18)$ \\
\hline \multicolumn{4}{|l|}{ Preoperative } \\
\hline Max-min, $\mu \mathrm{m}$ & $6.36 \pm 1.68$ & $5.47 \pm 2.45$ & $5.06 \pm 0.80$ \\
\hline $\mathrm{SD}^{\mathrm{b}}$ & $1.51 \pm 0.45$ & $1.27 \pm 0.68$ & $1.10 \pm 0.22^{c}$ \\
\hline \multicolumn{4}{|c|}{1 week postoperative } \\
\hline Max-min, $\mu \mathrm{m}^{\mathrm{b}}$ & $9.82 \pm 2.32$ & $8.82 \pm 3.13$ & $7.83 \pm 1.50^{\mathrm{c}}$ \\
\hline $\mathrm{SD}^{\mathrm{b}}$ & $2.39 \pm 0.72$ & $2.14 \pm 0.83$ & $1.76 \pm 0.29^{c}$ \\
\hline \multicolumn{4}{|c|}{1 month postoperative } \\
\hline Max-min, $\mu \mathrm{m}$ & $9.77 \pm 2.99$ & $9.71 \pm 3.58$ & $8.33 \pm 1.81$ \\
\hline SD & $2.42 \pm 0.71$ & $2.28 \pm 0.58$ & $1.90 \pm 0.37$ \\
\hline \multicolumn{4}{|c|}{3 months postoperative } \\
\hline Max-min, $\mu \mathrm{m}^{\mathrm{d}}$ & $10.77 \pm 2.69$ & $9.53 \pm 2.72$ & $8.11 \pm 1.57^{c}$ \\
\hline $\mathrm{SD}^{\mathrm{b}}$ & $2.58 \pm 0.64$ & $2.22 \pm 0.61$ & $2.04 \pm 0.41^{\mathrm{c}}$ \\
\hline \multicolumn{4}{|c|}{6 months postoperative } \\
\hline Max-min, $\mu \mathrm{m}^{\mathrm{b}}$ & $10.82 \pm 3.00$ & $9.76 \pm 3.49$ & $8.22 \pm 1.73^{\mathrm{c}}$ \\
\hline $\mathrm{SD}^{\mathrm{d}}$ & $2.70 \pm 0.75$ & $2.31 \pm 0.79$ & $1.96 \pm 0.34^{\mathrm{c}}$ \\
\hline
\end{tabular}

${ }^{\text {a }}$ Values expressed as mean $\pm \mathrm{SD}$. ${ }^{\mathrm{b}} \mathrm{P}<0.05$, comparison of three groups. ${ }^{\mathrm{c}} \mathrm{P}<0.05$ compared with group $\mathrm{A}$. ${ }^{\mathrm{d}} \mathrm{P}<0.01$, comparison of three groups. Two-way mixed-design ANOVA (Bonferroni's correction). Group A, non-wearing SCL; group B, wearing SCL $\leq 1$ year; group $\mathrm{C}=$ wearing $\mathrm{SCL}>1$ year; $\mathrm{SCL}$, soft contact lens; SD, standard deviation.

Table IV. Comparative data of corneal aberrations among three groups ${ }^{\mathrm{a}}$.

\begin{tabular}{|c|c|c|c|c|}
\hline Aberrations & Time & Group A $(n=22)$ & Group B $(n=17)$ & Group C $(n=18)$ \\
\hline \multirow[t]{4}{*}{ tHOA } & Preoperative $^{\mathrm{b}}$ & $0.38 \pm 0.10^{c}$ & $0.40 \pm 0.12$ & $0.51 \pm 0.15$ \\
\hline & 1 month postoperative ${ }^{\mathrm{d}}$ & $0.64 \pm 0.15^{\mathrm{c}, \mathrm{e}}$ & $0.69 \pm 0.17^{\mathrm{c}, \mathrm{e}}$ & $0.85 \pm 0.24^{\mathrm{e}}$ \\
\hline & 3 months postoperative ${ }^{b}$ & $0.65 \pm 0.15^{\mathrm{c}, \mathrm{e}}$ & $0.69 \pm 0.22^{\mathrm{c}, \mathrm{e}}$ & $0.78 \pm 0.20^{\mathrm{e}}$ \\
\hline & 6 months postoperative ${ }^{b}$ & $0.65 \pm 0.18^{\mathrm{c}, \mathrm{e}}$ & $0.68 \pm 0.22^{\mathrm{e}}$ & $0.76 \pm 0.18^{\mathrm{e}}$ \\
\hline \multirow[t]{4}{*}{ Spherical aberration } & Preoperative & $0.22 \pm 0.07$ & $0.19 \pm 0.08$ & $0.21 \pm 0.08$ \\
\hline & 1 month postoperative & $0.39 \pm 0.12^{\mathrm{e}}$ & $0.42 \pm 0.17^{\mathrm{e}}$ & $0.48 \pm 0.13^{\mathrm{e}}$ \\
\hline & 3 months postoperative ${ }^{b}$ & $0.37 \pm 0.10^{\mathrm{c}, \mathrm{e}}$ & $0.39 \pm 0.15^{\mathrm{e}}$ & $0.48 \pm 0.12^{\mathrm{e}}$ \\
\hline & 6 months postoperative ${ }^{b}$ & $0.38 \pm 0.12^{\mathrm{c}, \mathrm{e}}$ & $0.41 \pm 0.16^{\mathrm{e}}$ & $0.47 \pm 0.12^{\mathrm{e}}$ \\
\hline \multirow[t]{4}{*}{ Coma } & Preoperative $^{\mathrm{f}}$ & $0.18 \pm 0.07^{\mathrm{c}}$ & $0.23 \pm 0.12^{c}$ & $0.37 \pm 0.12$ \\
\hline & 1 month postoperative ${ }^{\mathrm{f}}$ & $0.35 \pm 0.17^{\mathrm{c}}$ & $0.38 \pm 0.21^{\mathrm{c}}$ & $0.61 \pm 0.22^{\mathrm{e}}$ \\
\hline & 3 months postoperative ${ }^{\mathrm{d}}$ & $0.36 \pm 0.15^{\mathrm{c}, \mathrm{e}}$ & $0.42 \pm 0.24^{\mathrm{c}}$ & $0.51 \pm 0.18^{\mathrm{e}}$ \\
\hline & 6 months postoperative ${ }^{\mathrm{d}}$ & $0.39 \pm 0.19^{c}$ & $0.42 \pm 0.21^{\mathrm{c}, \mathrm{e}}$ & $0.51 \pm 0.17^{\mathrm{e}}$ \\
\hline
\end{tabular}

${ }^{a}$ Values expressed as mean \pm standard deviation. ${ }^{b} \mathrm{P}<0.05$ comparison of three groups. ${ }^{\mathrm{c}} \mathrm{P}<0.05$, compared with group $\mathrm{C}$. ${ }^{\mathrm{d}} \mathrm{P}<0.01$ comparison of three groups. ${ }^{\mathrm{e}} \mathrm{P}<0.05$ postoperative vs. preoperative. ${ }^{\mathrm{f}} \mathrm{P}<0.001$ comparison of three groups. Two-way mixed-design ANOVA (Bonferroni's correction). Group A, non-wearing SCL; group B, wearing SCL $\leq 1$ year; group C, wearing SCL >1 year; tHOA, total high order aberration; SCL, soft contact lens.

can contribute to epithelial thinning (25). Finally, Efron (26) indicated that contact lens wear induced pathological changes in the corneal epithelium; in this study, mechanical stimulation was regarded as the main cause of corneal epithelial thinning. In summary, the hypoxia of the ocular surface and mechanical stimulation from the physical presence of contact lenses are the important causes of corneal epithelial thinning, which are related with the transmissibility of oxygen and the material of the lens $(9,22)$.
To the best of our knowledge, there have been no studies focusing on changes in corneal epithelial thickness following SCL wear after corneal refractive surgery. To recover the smooth corneal anterior surface, the corneal epithelium itself undergoes remodeling in response to corneal refractive surgery; this remodeling is characterized by uneven thickening of the corneal epithelium. The present results confirmed the findings of previous studies $(27,28)$. The corneal epithelium in Fig. 5 at all postoperative time 

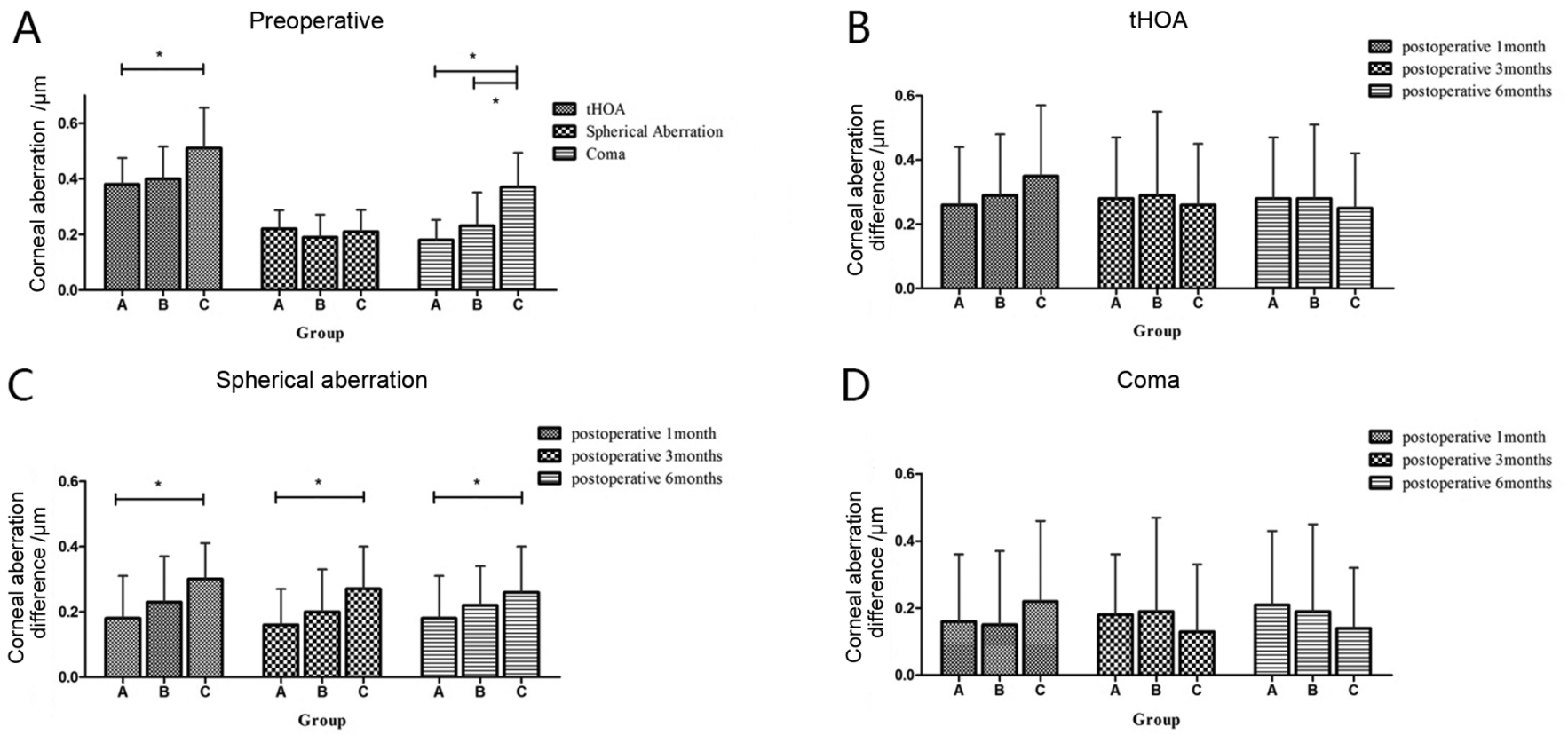

D

Coma

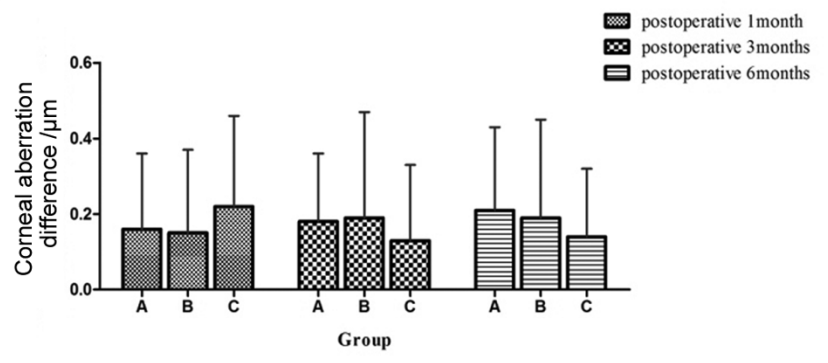

Figure 4. (A) Preoperative corneal aberrations of group A (non-SCL-wearing), group B (wearing SCL $\leq 1$ year) and group C (wearing SCL $>1$ year). (B) Total high order aberration difference, (C) spherical aberration difference and (D) coma difference of three groups at all postoperative time points. ${ }^{*} \mathrm{P}<0.05$. Two-way mixed ANOVA (Bonferroni's correction). SCL, soft contact lens; tHOA, total high order aberration.

points were in warmer colors compared with preoperative time points which means the corneal epithelium thickened following surgery. Previous studies demonstrated that the epithelium was stable 3 months after myopic surgery (29); therefore, the present study assessed the change in the epithelial thickness during the first 6 months after SMILE. Furthermore, the results demonstrated that the epithelial thicknesses of the central, nasal, inferonasal, inferior, inferotemporal and temporal sectors were thinner in the long-term wear group than in the non-wearing group at all postoperative time points; this was consistent with the preoperative results. There were no differences in the epithelial thicknesses of the superior, superonasal or superotemporal sectors among the three groups after surgery. In addition, the changes in epithelial thicknesses in the superior, superonasal and superotemporal segments were greater in the long-term wear group than in the non-wearing group. The changes in corneal epithelial thickness among the three groups before and after surgery were mainly due to the uneven regional thickening of the corneal epithelium postoperatively, possibly caused by the effects of pressure from the upper eyelid and SCL. In addition, previous studies have suggested that the epithelial thickness gradually recovers and reaches a stable state after a given period when contact lenses are no longer worn $(30,31)$; however, it cannot match the thickness in non-wearers (11). Although all patients in the present study had stopped contact lens wear 2 weeks preoperatively, the epithelial thickness was thinner in the wear group than that in the non-wearing group. The recovery of epithelial thickness after discontinuation of contact lens wear can also explain the difference in epithelial thicknesses before and after surgery.

No differences were found in terms of visual acuity or manifest refraction among the three groups at all postoperative time points after SMILE. The visual quality after SMILE did not appear to be affected by the thinning of the corneal epithelium induced by contact lens wear and corneal surgery. Considering that the postoperative epithelial healing response caused by SMILE is mild (32), changes in the corneal epithelium appear to have minimal effects on surgical outcomes. In a previous study, Lloyd-McKernan et al (33) reported that previous SCL wear had no negative impact on visual outcomes following LASIK and laser-assisted subepithelial keratecotomy (LASEK)/PRK when compared with a non-contact lens control group, which was consistent with the present results.

In the present study, the total higher-order and coma aberrations of the long-term wear group were greater than those of the non-wearing and short-term wear groups preoperatively, which was consistent with the results after surgery. By contrast, there were no significant differences in spherical aberrations among the three groups, while spherical aberrations at 3 and 6 months postoperatively were greater in the long-term wear group than in the non-wearing group. Consistent with this, the changes in spherical aberrations at 1,3 and 6 months postoperatively were greater in the long-term wear group than in the non-wearing group. Larger corneal aberrations observed in the long-term wear group may have been related to the influence of contact lenses on the normal physiology of the corneal epithelium (1). Long-term contact lens wear can cause a decrease of ocular surface glycocalyx, thereby inducing eye dryness and lowering of tear-film stability $(34,35)$. A decrease in tear-film stability can lead to greater corneal aberrations (36). In the present study, the tear-film stability and corneal epithelial integrity were better in the non-wearing group than in the long-term wear group, such that the corneal aberrations of the non-wearing group were smaller than those of the contact lens wear group. The total higher-order, spherical and coma aberrations of the three groups after SMILE were increased 


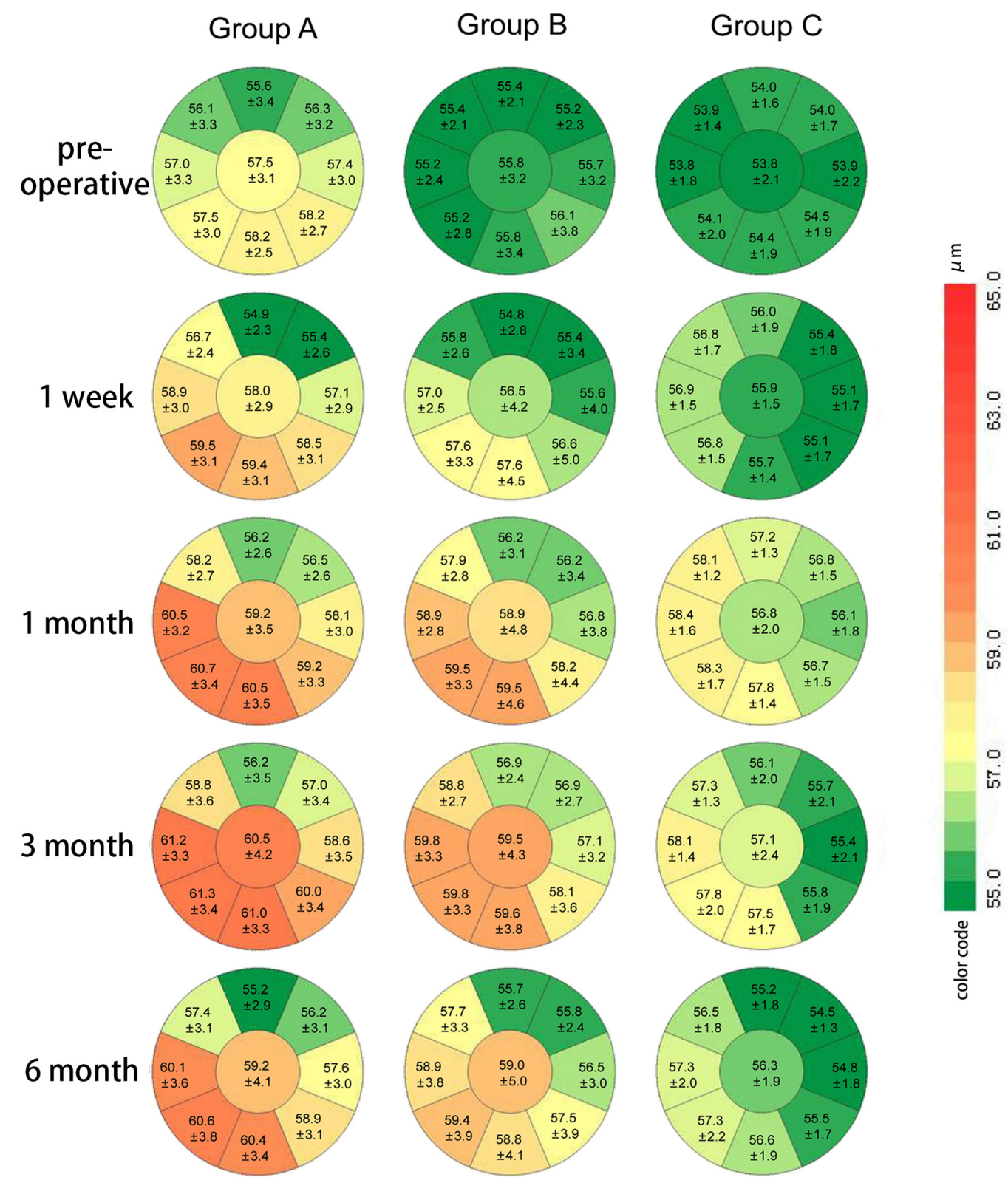

Figure 5. Nine sectors of corneal epithelial thickness of group A (non-SCL-wearing), group B (wearing SCL $\leq 1$ year) and group C (wearing SCL $>1$ year) for each pair of consecutive time points.

by different degrees. The total higher-order and coma aberrations of the three groups at all postoperative time points were consistent with the results before surgery, whereas the change in corneal aberrations was not statistically significant. This indicates that SMILE has a specific, but subtle influence on corneal aberrations, which may be related to the mild change in corneal shape and minimal or moderate changes in tear-film stability after SMILE.

The present study has a few limitations. Investigation of corneal epithelial thickness was limited to a region $5 \mathrm{~mm}$ in diameter. Furthermore, the thickness of the peripheral corneal epithelium may have been affected by the size of the palpebral fissure and the compression of the upper and lower eyelids on the cornea, which led to decreased accuracy and reliability of the measurement of peripheral corneal epithelial thickness and may have affected the comprehensive assessment of corneal epithelial thickness. With the possible development of more accurate high-resolution anterior segment optical coherence tomography technology, the epithelial thickness over the entire cornea can be studied to fully evaluate changes in epithelial thickness.

In summary, the present results indicate that long-term SCL wear will lead to epithelial thinning. However, uneven thickening of the corneal epithelium after SMILE and discontinuation of SCL wear do not impact visual acuity or manifest refraction after SMILE.

\section{Acknowledgements}

Not applicable.

\section{Funding}

No funding was received. 


\section{Availability of data and materials}

The datasets used and/or analyzed during the current study are available from the corresponding author on reasonable request.

\section{Authors' contributions}

YX, YW and XY made substantial contributions to acquisition of data and analysis of data. YW was involved in drafting the manuscript. YQ, BL and XZ made substantial contributions to conception and design, and revised the manuscript critically for important intellectual content. XZ and YX confirm the authenticity of all the raw data. All authors read and approved the final manuscript.

\section{Ethics approval and consent to participate}

Clinical trials registration reference ID: 2019-049 (The First Affiliated Hospital of Soochow University Institutional Review Board, Suzhou, China). This retrospective study was approved by the Ethics Committee of The First Affiliated Hospital of Soochow University, and all patients provided consent at the time of the surgery.

\section{Patient consent for publication}

Not applicable.

\section{Competing interests}

The authors declare that they have no competing interests.

\section{References}

1. Holden BA, Sweeney DF, Vannas A, Nilsson KT and Efron N Effects of long-term extended contact lens wear on the human cornea. Invest Ophthalmol Vis Sci 26: 1489-1501, 1985.

2. Liesegang TJ: Physiologic changes of the cornea with contact lens wear. CLAO J 28: 12-27, 2002.

3. Zhang X, Marchetti C, Lee J, Sun Y, Debanne S, Jiang Y, Kern J, Harrod M, Benetz BA, Pearlman E and Szczotka-Flynn L: The impact of lens care solutions on corneal epithelial changes during daily silicone hydrogel contact lens wear as measured by in vivo confocal microscopy. Contact Lens Anterior Eye 40: 33-41, 2017.

4. Yanai R, Ko JA, Morishige N, Chikama T, Ichijima $\mathrm{H}$ and Nishida T: Disruption of zonula occludens-1 localization in the rabbit corneal epithelium by contact lens-induced hypoxia. Invest Ophthalmol Vis Sci 50: 4605-4610, 2009.

5. Bastion ML and Mohamad MH: Study of the factors associated with the presence of white dots in the corneas of regular soft contact lens users from an Asian Country. Eye Contact Lens 32: 223-227, 2006

6. Edelhauser HF: The resiliency of the corneal endothelium to refractive and intraocular surgery. Cornea 19: 263-273, 2000

7. Yamamoto K, Ladage PM, Ren DH, Li L, Petroll WM, Jester JV and Cavanagh HD: Effect of eyelid closure and overnight contact lens wear on viability of surface epithelial cells in rabbit cornea. Cornea 21: 85-90, 2002

8. Hong J, Qian T, Yang Y, Jiang C, Liu Z, Sun X, Deng SX and $\mathrm{Xu}$ J: Corneal epithelial thickness map in long-term contact lenses wearers. Optom Vis Sci 91: 1455-1461, 2014.

9. Jalbert I, Sweeney DF and Stapleton F: The effect of long-term wear of soft lenses of low and high oxygen transmissibility on the corneal epithelium. Eye (Lond) 23: 1282-1287, 2009.

10. Stachura J, Mlyniuk P, Bloch W, Jimenez-Villar A, Grulkowski I and Kaluzny BJ: Shape of the anterior surface of the cornea after extended wear of silicone hydrogel soft contact lenses. Ophthalmic Physiol Opt 41: 683-690, 2021
11. Lei Y, Zheng X, Hou J, Xu B and Mu G: Effects of long-term soft contact lens wear on the corneal thickness and corneal epithelial thickness of myopic subjects. Mol Med Rep 11: 2020-2026, 2015.

12. Shehadeh-Mashor R, Mimouni M, Shapira Y, Sela T, Munzer G and Kaiserman I: Duration of contact lens removal before myopic refractive surgery. Eur J Ophthalmol 31: 1695-1699, 2021.

13. Sekundo W, Kunert KS and Blum M: Small incision corneal refractive surgery using the small incision lenticule extraction (SMILE) procedure for the correction of myopia and myopic astigmatism: Results of a 6 month prospective study. Br J Ophthalmol 95: 335-339, 2011.

14. Reinstein DZ, Archer TJ and Randleman JB: Mathematical model to compare the relative tensile strength of the cornea after PRK, LASIK, and small incision lenticule extraction. J Refract Surg 29: 454-460, 2013.

15. Denoyer A, Landman E, Trinh L, Faure JF, Auclin F and Baudouin C: Dry eye disease after refractive surgery: Comparative outcomes of small incision lenticule extraction versus LASIK. Ophthalmology 122: 669-676, 2015.

16. Ishii R, Shimizu K, Igarashi A, Kobashi $\mathrm{H}$ and Kamiya K: Influence of femtosecond lenticule extraction and small incision lenticule extraction on corneal nerve density and ocular surface: A 1-year prospective, confocal, microscopic study. J Refract Surg 31: 10-15, 2015.

17. Shah R, Shah S and Sengupta S: Results of small incision lenticule extraction: All-in-one femtosecond laser refractive surgery. J Cataract Refract Surg 37: 127-137, 2011.

18. Reinstein DZ, Archer TJ and Gobbe M: Lenticule thickness readout for small incision lenticule extraction compared to artemis three-dimensional very high-frequency digital ultrasound stromal measurements. J Refract Surg 30: 304-309, 2014.

19. Vestergaard AH, Grauslund J, Ivarsen AR and Hjortdal JØ: Central corneal sublayer pachymetry and biomechanical properties after refractive femtosecond lenticule extraction. J Refract Surg 30: 102-108, 2014.

20. Reinstein DZ, Archer TJ, Gobbe M, Silverman RH and Coleman DJ: Epithelial thickness in the normal cornea: Three-dimensional display with very high frequency ultrasound. J Refract Surg 24: 571-581, 2008.

21. Li Y, Tan O, Brass R, Weiss JL and Huang D: Corneal epithelial thickness mapping by Fourier-domain optical coherence tomography in normal and keratoconic eyes. Ophthalmology 119: 2425-2433, 2012

22. Pérez JG, Méijome JM, Jalbert I, Sweeney DF and Erickson P: Corneal epithelial thinning profile induced by long-term wear of hydrogel lenses. Cornea 22: 304-307, 2003.

23. Chen Y, Thompson DC, Koppaka V, Jester JV and Vasiliou V: Ocular aldehyde dehydrogenases: Protection against ultraviolet damage and maintenance of transparency for vision. Prog Retin Eye Res 33: 28-39, 2013.

24. Patel SV, McLaren JW, Hodge DO and Bourne WM: Confocal microscopy in vivo in corneas of long-term contact lens wearers. Invest Ophthalmol Vis Sci 43: 995-1003, 2002.

25. Markoulli M, Papas E, Cole N and Holden B: Corneal erosions in contact lens wear. Cont Lens Anterior Eye 35: 2-8, 2012.

26. Efron N: Contact lens-induced changes in the anterior eye as observed in vivo with the confocal microscope. Prog Retin Eye Res 26: 398-436, 2007.

27. Luft N, Ring MH, Dirisamer M, Mursch-Edlmayr AS, Kreutzer TC, Pretzl J, Bolz M and Priglinger SG: Corneal epithelial remodeling induced by small incision lenticule extraction (SMILE). Invest Ophthalmol Vis Sci 57: OCT176-OCT183, 2016.

28. Ryu IH, Kim BJ, Lee JH and Kim SW: Comparison of corneal epithelial remodeling after femtosecond laser-assisted LASIK and small incision lenticule extraction (SMILE). J Refract Surg 33: 250-256, 2017.

29. Reinstein DZ, Archer TJ and Gobbe M: Change in epithelial thickness profile $24 \mathrm{~h}$ and longitudinally for 1 year after myopic LASIK: Three-dimensional display with artemis very high-frequency digital ultrasound. J Refract Surg 28: 195-202, 2012.

30. Nourouzi H, Rajavi J and Okhovatpour MA: Time to resolution of corneal edema after longt-term contact lens wear. Am J Ophthalmol 142: 671-673, 2006.

31. Hashemi H, Firoozabadi MR, Mehravaran S and Gorouhi F: Corneal stability after discontinued soft contact lens wear. Cont Lens Anterior Eye 31: 122-125, 2008. 
32. Riau AK, Angunawela RI, Chaurasia SS, Lee WS, Tan DT and Mehta JS: Early corneal wound healing and inflammatory responses after refractive lenticule extraction (ReLEX). Invest Ophthalmol Vis Sci 52: 6213-6221, 2011.

33. Lloyd-McKernan A, Simo Mannion L and O'Dwyer V: The effect of previous soft contact lens wear on corneal refractive surgery outcomes. Cont Lens Anterior Eye 40: 301-310, 2017.

34. Fukui M, Yamada M, Akune Y, Shigeyasu C and Tsubota K: Fluorophotometric analysis of the ocular surface glycocalyx in soft contact lens wearers. Curr Eye Res 41: 9-14, 2016.
35. Guillon $M$ and Maissa C: Dry eye symptomatology of soft contact lens wearers and nonwearers. Optom Vis Sci 82: 829-834, 2005

36. Denoyer A, Rabut G and Baudouin C: Tear film aberration dynamics and vision-related quality of life in patients with dry eye disease. Ophthalmology 119: 1811-1818, 2012.

(i) (2) This work is licensed under a Creative Commons Attribution-NonCommercial-NoDerivatives 4.0 International (CC BY-NC-ND 4.0) License. 